\title{
Complex Behaviors of Epidemic Model with Nonlinear Rewiring Rate
}

\author{
Ding Fang, Yongxin Zhang, and Wendi Wang $(\mathbb{D}$ \\ Key Laboratory of Eco-Environments in Three Gorges Reservoir Region, School of Mathematics and Statistics, \\ Southwest University, Chongqing 400715, China
}

Correspondence should be addressed to Wendi Wang; wendi@swu.edu.cn

Received 21 November 2019; Revised 28 March 2020; Accepted 15 April 2020; Published 8 May 2020

Academic Editor: Átila Bueno

Copyright (c) 2020 Ding Fang et al. This is an open access article distributed under the Creative Commons Attribution License, which permits unrestricted use, distribution, and reproduction in any medium, provided the original work is properly cited.

An SIS propagation model with the nonlinear rewiring rate on an adaptive network is considered. It is found by bifurcation analysis that the model has the complex behaviors which include the transcritical bifurcation, saddle-node bifurcation, Hopf bifurcation, and Bogdanov-Takens bifurcation. Especially, a bifurcation curve with " $\mathrm{S}$ " shape emerges due to the nonlinear rewiring rate, which leads to multiple equilibria and twice saddle-node bifurcations. Numerical simulations show that the model admits a homoclinic bifurcation and a saddle-node bifurcation of the limit cycle.

\section{Introduction}

Mathematical models of epidemic propagations on networks have been proposed and studied by many papers (see [1-3] and the references cited therein) due to the importance in applications. Normile [4] described the network of SARS transmissions through hospitals and transportation. Zhang et al. [5, 6] modeled the transmission of H7N9 through transportation networks in China, respectively. Wang et al. [7-9] studied the impact of virus transmission on the network of mobile devices, respectively.

In recent years, there has been much interest in studying epidemic propagation on adaptive networks [10-15]. This is because behavior changes during the course of epidemic propagation are quite common and have the strong influence on the progression of disease. Indeed, an individual who links to an infective node tends to break the linkage and connect to a safer neighbor. For these reasons, Gross et al. [16] extended the classical SIS model in a static network to the adaptive network whose structure changes with the progression of epidemic disease. This model is important because it simulates the spread of epidemic disease in the network with the behavior changes and captures the key features of disease evolutions. There have been a number of studies about epidemic propagation on the adaptive network in this direction (see [11-13] and the references cited therein).

The following model, which originates from $[16,17]$, is presented in [18]:

$$
\left\{\begin{array}{l}
{[\dot{S}]=-\tau[\mathrm{SI}]+\gamma[I]} \\
{[\dot{I}]=\tau[\mathrm{SI}]-\gamma[I]} \\
{[\dot{\mathrm{SI}}]=-\gamma[\mathrm{SI}]+\gamma[\mathrm{II}]-\tau[\mathrm{SI}]-\tau[\mathrm{ISI}]+\tau[\mathrm{SSI}]-\omega[\mathrm{SI}]} \\
{[\dot{\mathrm{II}}]=-2 \gamma[\mathrm{II}]+2 \tau[\mathrm{SI}]+2 \tau[\mathrm{ISI}]} \\
{[\dot{\mathrm{SS}}]=2 \gamma[\mathrm{SI}]-2 \tau[\mathrm{SSI}]+2 \omega[\mathrm{SI}]}
\end{array}\right.
$$

where $[S]$ and $[I]$ denote the expected numbers of susceptible and infectious nodes, respectively. [SI] represents the expected number of SI edges, which means a susceptible node contacts with an infected node; [SS] and [II] have similar meanings. [SSI] and [ISI] denote the expected numbers of SSI and ISI triples [17]. An infected node can infect any susceptible neighbor at rate $\tau$ and recover to susceptible state at rate $\gamma$. The rewiring term is $\omega[\mathrm{SI}]$, which means that the susceptible individual disconnects with an infected neighbor at rate $\omega$ called as rewiring rate and randomly selects a susceptible individual to reconnect 
immediately. Juher et al. [15] analyzed the dynamics of model (1). Bodó and Simon [14] gave further mathematical analysis about the stability of equilibria and Hopf bifurcation for model (1). By numerical simulations, they found that the model has the complex behaviors such as the Bogdanov-Takens bifurcation and double limit cycles. A very important work was given by Zhang et al. [19] recently. They obtained the complete analytical conditions for bifurcations of the model that reveals the mechanism of various complex phenomena in model (1).

Notice that humans may take different behaviors as the risk of disease transmission varies [20, 21]. For example, during the SARS outbreak in 2003, the government and public opinion urged people to take protection measures by reducing infectious contacts, and people adopt the stronger responses when the reported cases were increased [22, 23]. Thus, social contacts tend to disconnect infectious nodes and turn to safe connections. These activities result in the rise of rewiring rate when the number of infectious individuals increases. For this reason, we modify the rewiring rate in model (1) from $\omega[\mathrm{SI}]$ to $\omega[\mathrm{SI}]+\widetilde{\beta}[I][\mathrm{SI}]$, where $\widetilde{\beta}$ is a nonnegative coefficient that describes the influence of infective number on the rewiring rate. Then, model (1) is extended to

$$
\left\{\begin{array}{l}
{[\dot{S}]=-\tau[\mathrm{SI}]+\gamma[I]} \\
{[\dot{I}]=\tau[\mathrm{SI}]-\gamma[I]} \\
{[\dot{\mathrm{SI}}]=-(\gamma+\tau)[\mathrm{SI}]+\gamma[\mathrm{II}]-\tau[\mathrm{ISI}]+\tau[\mathrm{SSI}]} \\
-(\omega+\tilde{\beta}[I])[\mathrm{SI}] \\
{[\dot{\mathrm{II}}]=-2 \gamma[\mathrm{II}]+2 \tau[\mathrm{SI}]+2 \tau[\mathrm{ISI}]} \\
{[\dot{\mathrm{SS}}]=2 \gamma[\mathrm{SI}]-2 \tau[\mathrm{SSI}]+2(\omega+\tilde{\beta}[I])[\mathrm{SI}]}
\end{array}\right.
$$

It is clear that model (2) extends model (1) by introducing the infection-dependent response coefficient $\tilde{\beta}$. This is important for a larger epidemic outbreak where human behaviors are crucially affected by the number of infected individuals, which is an index of infection risk.

The objective of this paper is to present the analytical analysis for bifurcations of model (2). We find that the model exhibits the more complicated behaviors due to the nonlinear rewiring rate. Besides the transcritical bifurcation, saddle-node bifurcation, Hopf bifurcation, and Bogdanov-Takens bifurcation in model (1), there exists a bifurcation curve with " $S$ " shape, which is attributed to the nonlinear rewiring rate and leads to multiple stable equilibria and twice saddle-node bifurcations. More importantly, the disease can persist even though the basic reproduction number is less than unity when the bifurcation at the disease-free equilibrium is forward. Moreover, we find a scenario where a stable periodic solution coexists with a stable equilibrium.

This paper is organized as follows. In Section 2, we simplify the model by the technique of moment closure and study the distribution of equilibrium points of the reduced model. The detailed analysis of stability of the steady states and bifurcations of the system is presented in Section 3. Numerical simulations are presented in Section 4 to reveal more interesting global behaviors of the model and illustrate the effect of adaptive rewiring on epidemic spread. The paper ends with discussions in Section 5.

\section{Model Reduction and Steady States}

Notice that the time evolutions of pairs in equation (2) depend on triples, which makes the analysis of model (2) impossible. A common method is to use the moment closure technique to obtain the closed form of these equations. Here, we follow Zhang et al. [19] to assume the degree distribution of the network obeys the Poisson distribution. In this case, the triples can be approximated by pairs and singles according to Keeling [24]:

$$
\begin{aligned}
& {[\mathrm{SSI}]=\frac{[\mathrm{SS}][\mathrm{SI}]}{[S]},} \\
& {[\mathrm{ISI}]=\frac{[\mathrm{SI}][\mathrm{SI}]}{[S]} .}
\end{aligned}
$$

The first equality in (3) means that SSI links are determined by the product of number of SS links and the probability that a given susceptible node has an SI link. The second equality in (3) has the similar meaning.

Let $N$ be the number of vertices of the network and $n$ be the average degree of the graph. Since the derivative $2[\mathrm{SI}]+$ $[\mathrm{SS}]+[\mathrm{II}]$ equals zero, we have $2[\mathrm{SI}]+[\mathrm{SS}]+[\mathrm{II}]=n N$. Then, let us nondimensionalize the system by the transformations

$$
\begin{gathered}
P_{S}=\frac{[S]}{N}, \\
P_{I}=\frac{[I]}{N}, \\
P_{\mathrm{SI}}=\frac{2[\mathrm{SI}]}{n N}, \\
P_{\mathrm{SS}}=\frac{[\mathrm{SS}]}{n N}, \\
P_{\mathrm{II}}=\frac{[\mathrm{II}]}{n N},
\end{gathered}
$$

to obtain

$$
\left\{\begin{array}{l}
\dot{P}_{I}=\frac{\tau n}{2} P_{\mathrm{SI}}-\gamma P_{I}, \\
\dot{P}_{\mathrm{SI}}=-(\gamma+\tau+\omega) P_{\mathrm{SI}}+2 \gamma\left(1-P_{\mathrm{SI}}-P_{\mathrm{SS}}\right)+\tau n \\
\cdot\left(P_{\mathrm{SS}}-\frac{P_{\mathrm{SI}}}{2}\right) \frac{P_{\mathrm{SI}}}{1-P_{I}}-\beta P_{I} P_{\mathrm{SI}}, \\
\dot{P}_{\mathrm{SS}}=\gamma P_{\mathrm{SI}}-\tau n \frac{P_{\mathrm{SS}} P_{\mathrm{SI}}}{1-P_{I}}+\omega P_{\mathrm{SI}}+\beta P_{I} P_{\mathrm{SI}},
\end{array}\right.
$$

in which, the identities of $P_{S}+P_{I}=1$ and $P_{\mathrm{SI}}+P_{\mathrm{SS}}+P_{\mathrm{II}}=1$ are used, and $\beta=\widetilde{\beta} N$. In view of biological background, we 
assume that the parameters $\tau, \gamma$, and $n$ are positive constants, and the parameters $\omega$ and $\beta$ are nonnegative constants. It is easy to verify the domain

$$
\Omega=\left\{\left(P_{I}, P_{\mathrm{SI}}, P_{\mathrm{SS}}\right) \mid 0 \leq P_{\mathrm{SI}}, 0 \leq P_{\mathrm{SS}}, 0 \leq P_{I}<1, P_{\mathrm{SI}}+P_{\mathrm{SS}} \leq\right\} 1 .
$$

is a positive invariant set of system (5). In the rest of this paper, we study the dynamical behaviors of the system in $\Omega$.

The disease-free steady state (DEF) of system (5) is $E_{0}=(0,0,1)$. With the help of the computation method in [25], we get the basic reproduction number:

$$
R_{0}=\frac{\tau n}{(\gamma+\omega)}
$$

The endemic steady states are determined by

$$
\begin{gathered}
0=\frac{\tau n}{2} P_{\mathrm{SI}}-\gamma P_{I}, \\
0=-\gamma P_{\mathrm{SI}}+2 \gamma\left(1-P_{\mathrm{SI}}-P_{\mathrm{SS}}\right)-\tau P_{\mathrm{SI}}-\frac{\tau n}{2} \frac{P_{\mathrm{SI}} P_{\mathrm{SI}}}{1-P_{I}} \\
+\tau n \frac{P_{\mathrm{SS}} P_{\mathrm{SI}}}{1-P_{I}}-\omega P_{\mathrm{SI}}-\beta P_{I} P_{\mathrm{SI}}, \\
0=\gamma P_{\mathrm{SI}}-\tau n \frac{P_{\mathrm{SS}} P_{\mathrm{SI}}}{1-P_{I}}+\omega P_{\mathrm{SI}}+\beta P_{I} P_{\mathrm{SI}} .
\end{gathered}
$$

Obviously, from equations (8) and (10), we have

$$
\begin{aligned}
& P_{\text {SI }}=\frac{2 \gamma P_{I}}{\tau n}, \\
& P_{\text {SS }}=\frac{-\beta}{\tau n} P_{I}^{2}+\frac{-\gamma-\omega+\beta}{\tau n} P_{I}+\frac{\gamma+\omega}{\tau n} .
\end{aligned}
$$

By substituting these expressions into equation (9), we can deduce $P_{I}$ which satisfies the following equation:

$$
F\left(P_{I}\right):=\mathscr{A} P_{I}^{3}+\mathscr{B} P_{I}^{2}+\mathscr{C} P_{I}+\mathscr{D}=0,
$$

where

$$
\begin{aligned}
& \mathscr{A}=\beta, \\
& \mathscr{B}=\omega-2 \beta-\tau, \\
& \mathscr{C}=-2 \omega+\beta+\tau+\tau n, \\
& \mathscr{D}=(\gamma+\omega)\left(1-R_{0}\right) .
\end{aligned}
$$

Set

$$
\begin{aligned}
& A=\mathscr{B}^{2}-3 \mathscr{A} c, \\
& B=\mathscr{B} \mathscr{C}-9 \mathscr{A} \mathscr{D}, \\
& C=\mathscr{C}^{2}-3 \mathscr{B} \mathscr{D} .
\end{aligned}
$$

Motivated by [26], we define the discriminant of the cubic function $F\left(P_{I}\right)$ by

$$
\Delta_{0}:=B^{2}-4 A C \text {. }
$$

Notice that $A \geq 0$ if $\Delta_{0}=0$. Similar to [26], we see that the positive roots of equation (12) are classified by the signs of
$\Delta_{0}, \mathscr{B}, \mathscr{C}$, and $\mathscr{D}$. Then, we state the distribution of equilibria of equation (12) in three cases.

$$
\mathrm{C} 1: R_{0}>1 \text { : }
$$

(1) If $\mathscr{C} \leq 0$, then $F\left(P_{I}\right)$ has a unique positive root.

(2) Suppose that $\mathscr{C}>0$ and $\mathscr{B} \geq 0$. Then, $F\left(P_{I}\right)$ has a unique positive root.

(3) Suppose that $\mathscr{C}>0$ and $\mathscr{B}<0$. Then, $F\left(P_{I}\right)$ has a unique positive root if $\Delta_{0}>0$, has a positive root of multiplicity 2 and another positive root if $\Delta_{0}=0$ and $A>0$, has a positive root of multiplicity 3 if $\Delta_{0}=A=0$, and has three distinct positive roots if $\Delta_{0}<0$

C2: $R_{0}<1$ :

(1) Suppose that $\mathscr{C} \geq 0$ and $\mathscr{B} \geq 0$. Then, $F\left(P_{I}\right)$ has no positive root.

(2) Suppose that $\mathscr{C} \geq 0$ and $\mathscr{B}<0$. Then, $F\left(P_{I}\right)$ has no positive root if $\Delta_{0}>0$, has a positive root of multiplicity 2 if $\Delta_{0}=0$, and has two positive roots if $\Delta_{0}<0$.

(3) Suppose that $\mathscr{C}<0$. Then, $F\left(P_{I}\right)$ has no positive root if $\Delta_{0}>0$, has a positive root of multiplicity 2 if $\Delta_{0}=0$, and has two positive roots if $\Delta_{0}<0$.

C3: $R_{0}=1$ :

(1) Suppose that $\mathscr{C}<0$. Then, $F\left(P_{I}\right)$ has a unique positive root.

(2) Suppose that $\mathscr{C}=0$ and $\mathscr{B}<0$. Then, $F\left(P_{I}\right)$ has a unique positive root.

(3) Suppose that $\mathscr{C} \geq 0$ and $\mathscr{B} \geq 0$. Then, $F\left(P_{I}\right)$ has no positive root.

(4) Suppose that $\mathscr{C}>0$ and $\mathscr{B}<0$. Then, $F\left(P_{I}\right)$ has no positive root if $\Delta_{0}>0$, has a positive root of multiplicity 2 if $\Delta_{0}=0$, and has two positive roots if $\Delta_{0}<0$

Based on the above analysis about the roots of equation (12), we obtain the distributions of endemic equilibria for system (5), which are shown in Table 1. It is obvious that the distributions of the endemic equilibria are complex, which means that there exist rich bifurcations in system (5). We will elucidate these bifurcations in Section 3.

\section{Stability and Bifurcation Analysis}

In this section, we study the stability of steady states to get the sufficient and necessary conditions of backward bifurcation and Hopf bifurcation. Besides, we show that the system undergoes the Bogdanov-Takens bifurcation.

3.1. Backward Bifurcation. For the stability of the diseasefree steady state (DEF), we have the following proposition.

Proposition 1. The DEF is asymptotically stable if $R_{0}<1$ and is unstable if $R_{0}>1$. 
TABLE. 1: Distribution of the endemic equilibria.

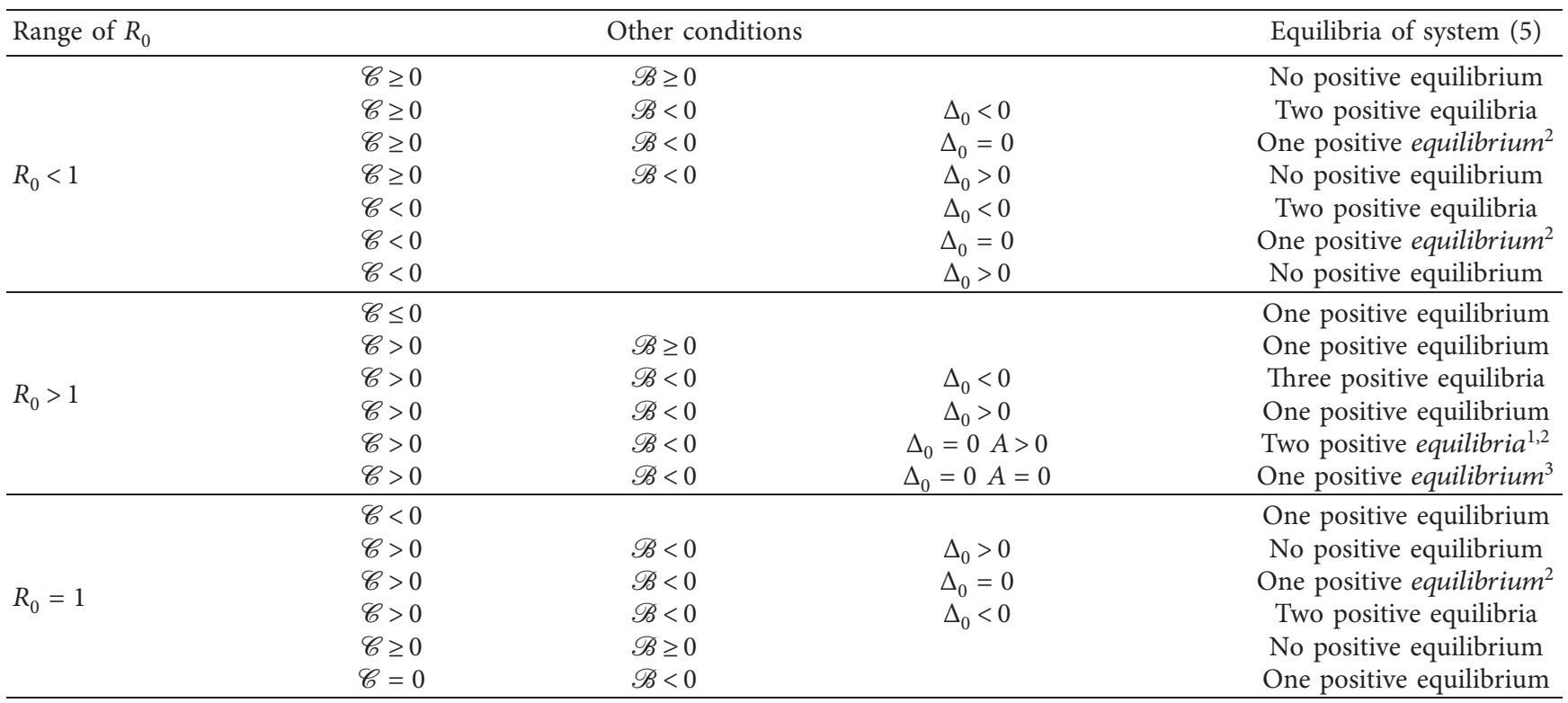

Two positive equilibria ${ }^{1,2}$ mean that there are a simple equilibrium and another equilibrium of multiplicity 2 . One positive equilibrium ${ }^{2}$ means that there is an equilibrium of multiplicity 2 . One positive equilibrium ${ }^{3}$ means that there is an equilibrium of multiplicity 3 . The remaining cases represent a simple equilibrium.

Proof. It is easy to find that $-\gamma$ is an eigenvalue of the Jacobian matrix at DEF. The other two eigenvalues $\lambda_{1}$ and $\lambda_{2}$ are the roots of

$$
\lambda^{2}+\lambda(3 \gamma-\tau n+\omega+\tau)+2 \gamma(\gamma-\tau n+\omega)=0 .
$$

If $R_{0}<1$, then $\tau n<\gamma+\omega$, which leads to $3 \gamma-\tau n+\omega+\tau>0$. Thus, both $\lambda_{1}$ and $\lambda_{2}$ have negative real parts, and therefore, the DEF is asymptotically stable. If $R_{0}>1$, then one root of (16) is positive, which means that the DEF is unstable.

There exist two endemic steady states when $R_{0}<1$. This implies the possibility that the system has a backward bifurcation. Let us now figure out the conditions for the backward bifurcation to appear. Set

$$
\begin{aligned}
& a_{0}=2 \gamma n(\gamma+\omega)\left(\frac{-\beta+\gamma+\omega}{(2 n+1) \gamma+\omega} n-1\right), \\
& b_{0}=\frac{2 \gamma n^{2}}{2 \gamma n+\gamma+\omega} .
\end{aligned}
$$

By the technique of central manifold [27], we obtain the following theorem.

Theorem 1. System (5) exhibits a backward bifurcation at $D E F$ when $R_{0}$ crosses unity if $a_{0}>0$ and a forward bifurcation if $a_{0}<0$.

The proof of Theorem 1 is deferred to Appendix A. It is noting that the coefficient $\beta$ has a large impact on the backward bifurcation although it has no effect on $R_{0}$. From Table 1, we find that the system may undergo a saddle-node bifurcation when the two equilibria coalesce into one equilibrium. Thus, we derive the conditions for the saddlenode bifurcation. Interestingly, we show that the system has a forward bifurcation curve that looks like "S" shape.

3.2. Saddle-Node and Hopf Bifurcations. In this section, we first present the conditions that system (5) undergoes a saddle-node bifurcation. The Jacobian matrix of the system at an endemic state $E^{*}=\left(P_{I}^{*}, P_{\mathrm{SI}}^{*}, P_{\mathrm{SS}}^{*}\right)$ is

$$
J\left(E^{*}\right)=\left(\begin{array}{ccc}
-\gamma & \frac{\tau n}{2} & 0 \\
2 \gamma P_{I}^{*} \frac{-2 \beta P_{I}^{* 2}+(3 \beta-\omega-2 \gamma) P_{I}^{*}-\beta+\omega+\gamma}{\tau n\left(1-P_{I}^{*}\right)^{2}} & -\frac{2 \gamma P_{I}^{*}}{1-P_{I}^{*}}-\tau-2 \gamma & 2 \gamma\left(\frac{P_{I}^{*}}{1-P_{I}^{*}}-1\right.
\end{array}\right) .
$$


The eigenvalues of $J\left(E^{*}\right)$ are the roots of

$$
\lambda^{3}+G_{2}\left(P_{I}^{*}\right) \lambda^{2}+G_{1}\left(P_{I}^{*}\right) \lambda+G_{0}\left(P_{I}^{*}\right)=0,
$$

where

$G_{2}\left(P_{I}^{*}\right)=-\frac{\gamma P_{I}^{*}-\tau P_{I}^{*}+3 \gamma+\tau}{-1+P_{I}^{*}}=\frac{\gamma P_{I}^{*}+\tau\left(1-P_{I}^{*}\right)+3 \gamma}{1-P_{I}^{*}}>0$,

$G_{1}\left(P_{I}^{*}\right)=\frac{\gamma\left[2 \beta P_{I}^{* 3}+(-3 \beta+\omega-\tau) P_{I}^{*^{2}}+(\beta+3 \gamma-\omega) P_{I}^{*}+2 \gamma+\tau\right]}{\left(-1+P_{I}^{*}\right)^{2}}$,

$G_{0}\left(P_{I}^{*}\right)=\frac{2 \gamma^{2} P_{I}^{*} F^{\prime}\left(P_{I}^{*}\right)}{\left(1-P_{I}^{*}\right)^{2}}$

Thus, the matrix $J\left(E^{*}\right)$ admits a zero eigenvalue if and only if $G_{0}\left(P_{I}^{*}\right)=0$, i.e., $F^{\prime}\left(P_{I}^{*}\right)=0$. Furthermore, zero is a simple eigenvalue if $G_{1}\left(P_{I}^{*}\right) \neq 0$, and Table 1 shows that $E^{*}$ is an endemic equilibrium of multiplicity 2 or 3 when $\Delta_{0}=0$. Consequently, we can state the following theorem.

Theorem 2. Assume $\Delta_{0}=0$. Then, system (5) has an endemic equilibrium $\widehat{E}=\left(\widehat{P}_{I}, \widehat{P}_{S I}, \widehat{P}_{S S}\right)$ of multiplicity 2 if one of the following is satisfied:

(a) $R_{0}<1, \mathscr{C} \geq 0$, and $\mathscr{B}<0$

(b) $R_{0}<1$ and $\mathscr{C}<0$

(c) $R_{0}>1, \mathscr{C}>0, \mathscr{B}<0$, and $A>0$

(d) $R_{0}=1, \mathscr{C}>0$, and $\mathscr{B}<0$

Furthermore, system (5) undergoes a saddle-node bifurcation at $\widehat{E}$ if $G_{1}\left(\widehat{P}_{I}\right) \neq 0$.

The proof of Theorem 2 is omitted because it is similar to that in $[19,26,28]$.

From Theorems 1 and 2 , we find that the rewiring rate $\beta$ has remarkable impact on behaviors of system (5). To illustrate this, we depict the bifurcation diagrams of system (5) with different $\beta$ in Figure 1, where the basal parameters are $\gamma=0.1, n=6, \omega=2$.9. In Figure 1 , when the rewiring rate $\beta$ increases from zero, the type of bifurcation changes. For $0<\beta<2.3$, the bifurcation at DEF is backward, and there exists a saddle-node bifurcation SN (see the solid bifurcation curve of Figure 1). At $\beta=2.3$, which leads the critical condition $a_{0}=0$, the DEF becomes an equilibrium of multiplicity 3 (see the dashed bifurcation curve of Figure 1). As $\beta$ increases further, the bifurcation at DEF becomes forward, and there exist two saddle-node bifurcations $\mathrm{SN}_{1}$ and $\mathrm{SN}_{2}$ for $\beta>2.3$. It is notable that the equilibrium $\mathrm{SN}_{2}$ coincides with DEF at $\beta=2.3$ if $R_{0}=1$. In a word, we find that the coefficient $\beta$ can induce the bifurcation curve which looks like "S" shape (see the dotted dash bifurcation curve and dotted bifurcation curve of Figure 1). By Theorems 1 and 2 , when $\beta>2.3$, the bifurcation curve will remain $\mathrm{S}$ shaped. More importantly, the disease can persist even though $R_{0}<1$ when the bifurcation at DEF is forward (see the dotted dash bifurcation curve of Figure 1).

By (21), we know $G_{2}\left(P_{I}^{*}\right)>0$. Using the Routh-Hurwitz criterion and invoking the method to examine the Hopf bifurcation in [29], we see that the equilibrium point $\left(P_{I}^{*}, P_{\text {SI }}^{*}, P_{\text {SS }}^{*}\right)$ is stable if

$$
\begin{aligned}
G_{0}\left(P_{I}^{*}\right) & >0, \\
G_{2}\left(P_{I}^{*}\right) G_{1}\left(P_{I}^{*}\right)-G_{0}\left(P_{I}^{*}\right) & >0 .
\end{aligned}
$$

Moreover, the Hopf bifurcation occurs when

$$
\begin{aligned}
G_{2}\left(P_{I}^{*}\right) G_{1}\left(P_{I}^{*}\right)-G_{0}\left(P_{I}^{*}\right) & =0, \\
G_{1}\left(P_{I}^{*}\right) & >0 .
\end{aligned}
$$

3.3. Bogdanov-Takens Bifurcation. When $\Delta_{0}=0$ and one of the conditions (a), (b), (c), and (d) in Theorem 2 is satisfied, system (5) has an endemic equilibrium $\widehat{E}=\left(\widehat{P}_{I}, \widehat{P}_{\mathrm{SI}}, \widehat{P}_{\mathrm{SS}}\right)$ of multiplicity 2 . It is easy to verify $A \neq 0$ under these conditions. Furthermore, we see that the components of $\widehat{E}$ are given by

$$
\begin{aligned}
\widehat{P}_{I} & =-\frac{B}{2 A}, \\
\widehat{P}_{\mathrm{SI}} & =-\frac{\gamma B}{\tau n A}, \\
\widehat{P}_{\mathrm{SS}} & =\frac{-\beta B^{2}}{4 \tau n A^{2}}+\frac{\gamma+\omega-\beta}{2 \tau n A} B+\frac{\gamma+\omega}{\tau n} .
\end{aligned}
$$

If $G_{1}\left(\widehat{P}_{I}\right) \neq 0$, by Theorem $2, \widehat{E}$ is a saddle node. If $G_{1}\left(\widehat{P}_{I}\right)=0$, it is obvious that the eigenvalues of the Jacobian matrix at $\widehat{E}$ are $0,0,-G_{2}\left(\widehat{P}_{I}\right)<0$. We show in the following $\widehat{E}$ is a Bogdanov-Takens point.

Theorem 3. Assume one of the conditions (a), (b), (c), and (d) in Theorem 2 is satisfied. If $\Delta_{0}=0$ and $G_{1}\left(\widehat{P}_{I}\right)=0$, then $\widehat{E}$ is a Bogdanov-Takens point, and system (5) localized at $\widehat{E}$ is topologically equivalent to

$$
\left\{\begin{array}{l}
\dot{\mu}=\nu \\
\dot{\nu}=\mu^{2}+\mu \nu+\mathcal{O}\left(|\mu, \nu|^{3}\right) .
\end{array}\right.
$$

Proof. We just outline the main steps and leave the cumbersome details in Appendix B. First, let us bring $\widehat{E}$ to the origin by substituting $x=P_{I}-\widehat{P}_{I}, y=P_{\mathrm{SI}}-\widehat{P}_{\mathrm{SI}}, z=$ $P_{\mathrm{SS}}-\widehat{P}_{\mathrm{SS}}$. Then, system (5) becomes 


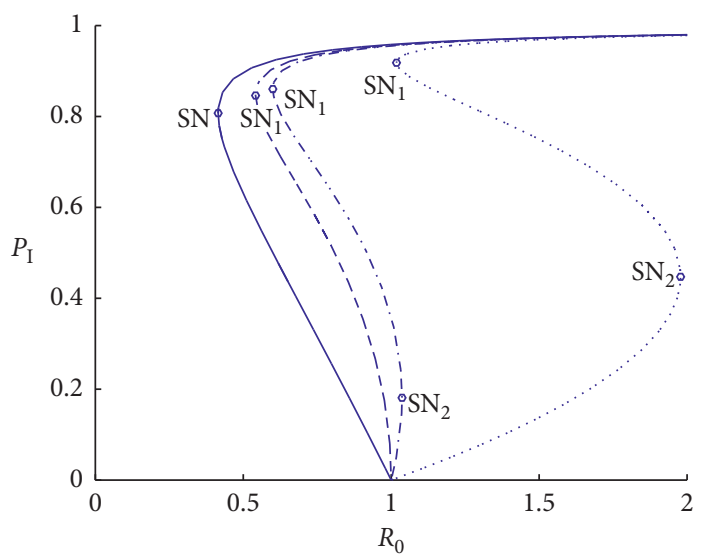

FIGURE 1: Bifurcation diagrams of system (5) where $\mathrm{SN}, \mathrm{SN}_{1}$, and $\mathrm{SN}_{2}$ represent saddle-node points. The parameter values are $\gamma=0.1, n=6$, $\omega=2.9$, and $\beta=0$ (solid), 2.3 (dashed), 3.5 (dotted dash), or 15 (dotted).

$$
\begin{aligned}
& \int \dot{x}=-\gamma x+\frac{\tau n}{2} y, \\
& \dot{y}=2 \frac{\gamma \widehat{P}_{I}}{\tau n}\left(\frac{\beta \widehat{P}_{I}+\gamma+\omega}{1-\widehat{P}_{I}}-\frac{\gamma \widehat{P}_{I}}{\left(1-\widehat{P}_{I}\right)^{2}}-\beta\right) x+\left(\frac{-2 \gamma \widehat{P}_{I}}{1-\widehat{P}_{I}}-\tau-2 \gamma\right) y+2 \gamma\left(\frac{\widehat{P}_{I}}{1-\widehat{P}_{I}}-1\right) z \\
& -\frac{\tau n \widehat{P}_{\mathrm{SI}}\left(\widehat{P}_{\mathrm{SI}}-2 \widehat{P}_{\mathrm{SS}}\right)}{2\left(1-\widehat{P}_{I}\right)^{3}} x^{2}-\frac{\beta \widehat{P}_{I}^{2}+\tau n \widehat{P}_{\mathrm{SI}}-\tau n \widehat{P}_{\mathrm{SS}}-2 \beta \widehat{P}_{I}+\beta}{\left(1-\widehat{P}_{I}\right)^{2}} x y+\frac{\tau n \widehat{P}_{\mathrm{SI}}}{\left(1-\widehat{P}_{I}\right)^{2}} x z-\frac{\tau n}{2\left(1-\widehat{P}_{I}\right)} y^{2}+\frac{\tau n}{1-\widehat{P}_{I}} y z+\mathcal{O}\left(|x, y, z|^{3}\right), \\
& \dot{z}=2 \frac{\gamma \widehat{P}_{I}}{\tau n}\left(-\frac{\beta \widehat{P}_{I}+\gamma+\omega}{1-\widehat{P}_{I}}+\beta\right) x-2 \frac{\gamma \widehat{P}_{I}}{1-\widehat{P}_{I}} z-\frac{\tau n \widehat{P}_{\mathrm{SS}} \widehat{P}_{\mathrm{SI}}}{\left(1-\widehat{P}_{I}\right)^{3}} x^{2}+\frac{\beta \widehat{P}_{I}^{2}-\tau n \widehat{P}_{\mathrm{SS}}-2 \beta \widehat{P}_{I}+\beta}{\left(1-\widehat{P}_{I}\right)^{2}} x y-\frac{\tau n \widehat{P}_{\mathrm{SI}}}{\left(1-\widehat{P}_{I}\right)^{2}} x z-\frac{\tau n}{1-\widehat{P}_{I}} y z+\mathcal{O}\left(|x, y, z|^{3}\right) .
\end{aligned}
$$

Because $\widehat{E}$ is an equilibrium of multiplicity of 2 , we can get $G_{0}\left(\widehat{P}_{I}\right)=0$ from (21). From (19), we see that the Jacobian matrix at $\widehat{E}=\left(\widehat{P}_{I}, \widehat{P}_{\text {SI }}, \widehat{P}_{\text {SS }}\right)$ has three eigenvalues: $\lambda_{1}=\lambda_{2}=$ 0 and $\lambda_{3}=-G_{2}\left(\widehat{P}_{I}\right)<0$ if $G_{1}\left(\widehat{P}_{I}\right)=0$. The generalized eigenvectors corresponding to 0 are

$$
\begin{aligned}
& V_{1}=\left(-\frac{\tau n}{\gamma+\left(2 \widehat{P}_{I}-1\right) \beta+\omega},-\frac{2 \gamma}{\gamma+\left(2 \widehat{P}_{I}-1\right) \beta+\omega}, 1\right)^{\prime}, \\
& V_{2}=\left(\frac{\tau n}{\left(2 \beta \widehat{P}_{I}-\beta+\gamma+\omega\right) \gamma}, 0,-\frac{\widehat{P}_{I}+1}{2 \gamma \widehat{P}_{I}}\right)^{\prime},
\end{aligned}
$$

\section{and the eigenvectors corresponding to $\lambda_{3}$ are}

$$
V_{3}=\left(-\frac{\tau n\left((\tau+\gamma) \widehat{P}_{I}-\tau-3 \gamma\right)}{2 \gamma \widehat{P}_{I}\left(2 \beta \widehat{P}_{I}-\beta+\gamma+\omega\right)}, \frac{\left((\tau+\gamma) \widehat{P}_{I}-\tau-3 \gamma\right)\left((\tau-2 \gamma) \widehat{P}_{I}-\tau-2 \gamma\right)}{\gamma \widehat{P}_{I}\left(2 \beta \widehat{P}_{I}-\beta+\gamma+\omega\right)\left(\widehat{P}_{I}-1\right)}, 1\right)^{\prime}
$$


Here, the symbol ' means the transpose of a vector. Then, the matrix $V=\left(V_{1}, V_{2}, V_{3}\right)$ is used for the nonsingular linear transformation:

$$
\left(\begin{array}{l}
x \\
y \\
z
\end{array}\right)=V\left(\begin{array}{l}
\mu \\
\nu \\
\xi
\end{array}\right)
$$

As a result, we get

$$
\left\{\begin{array}{l}
\dot{\mu}=\nu+L_{20} \mu^{2}+L_{11} \mu \nu+L_{02} \nu^{2}+\xi \cdot \mathcal{O}(|\mu, \nu, \xi|)+\mathcal{O}\left(|\mu, \nu, \xi|^{3}\right), \\
\dot{\nu}=M_{20} \mu^{2}+M_{11} \mu \nu+M_{02} \nu^{2}+\xi \cdot \mathcal{O}(|\mu, \nu, \xi|)+\mathcal{O}\left(|\mu, \nu, \xi|^{3}\right), \\
\dot{\xi}=-G_{2}\left(\widehat{P}_{I}\right) \xi+\mathcal{O}\left(|\mu, \nu, \xi|^{2}\right) .
\end{array}\right.
$$

The expressions of the coefficients are shown in Appendix B. By the center manifold theorem [30], system (31) restricted to the center manifold becomes

$$
\left\{\begin{array}{l}
\dot{\mu}=\nu+L_{20} \mu^{2}+L_{11} \mu \nu+L_{02} \nu^{2}+\mathcal{O}\left(|\mu, \nu|^{3}\right), \\
\dot{v}=M_{20} \mu^{2}+M_{11} \mu \nu+M_{02} \nu^{2}+\mathcal{O}\left(|\mu, \nu|^{3}\right) .
\end{array}\right.
$$

We use the near-identity transformation:

$$
\begin{aligned}
& \mu=X+\frac{1}{2}\left(L_{11}+M_{02}\right) X^{2}+L_{02} X Y+\mathcal{O}\left(|X, Y|^{3}\right), \\
& \nu=Y-L_{20} X^{2}+M_{02} X Y+\mathcal{O}\left(|X, Y|^{3}\right),
\end{aligned}
$$

and then rewrite $X, Y$ into $\mu$ and $\nu$ to get

$$
\left\{\begin{array}{l}
\dot{\mu}=v \\
\dot{\nu}=M_{20} \mu^{2}+\left(M_{11}+2 L_{20}\right) \mu \nu+\mathcal{O}\left(|\mu, v|^{3}\right) .
\end{array}\right.
$$

It is proved in Appendix $\mathrm{B}$ that $M_{20}>0$ and $M_{11}+2 L_{20}>0$. If we rescale the time and variables by

$$
(\mu, v, t) \longrightarrow\left(\frac{M_{20}}{\left(M_{11}+2 L_{20}\right)^{2}} \mu, \frac{M_{20}^{2}}{\left(M_{11}+2 L_{20}\right)^{3}} \nu, \frac{M_{11}+2 L_{20}}{M_{20}} t\right),
$$

then system (34) is topologically equivalent to normal form (26).

From Theorem 3, we need two parameters to unfold the codimension two singularity of $\widehat{E}$. Firstly, let $\left(\tau, \omega, n, \gamma_{1}, \beta_{1}\right)$ satisfy the conditions in Theorem 3 . Then, we have

$$
\left\{\begin{array}{l}
\beta_{1}=\frac{(-3 \omega+3 \tau) \widehat{P}_{I}^{2}+2 \omega-\tau}{\left(2 \widehat{P}_{I}-1\right)\left(3 \widehat{P}_{I}^{2}-2\right)}, \\
\gamma_{1}=\frac{\tau\left(\widehat{P}_{I}-1\right)^{2}}{3 \widehat{P}_{I}^{2}-2} .
\end{array}\right.
$$

In the following, we consider system (5) for parameters $(\beta, \gamma)$ in a neighborhood $\left(\beta_{1}, \gamma_{1}\right)$. Substituting

$$
\begin{aligned}
& \beta=\beta_{1}+\varepsilon_{1}, \\
& \gamma=\gamma_{1}+\varepsilon_{2},
\end{aligned}
$$

into system (5), we obtain a perturbed system

$$
\left\{\begin{array}{l}
\dot{P}_{I}=\frac{\tau n}{2} P_{\mathrm{SI}}-\left(\gamma_{1}+\varepsilon_{2}\right) P_{I} \\
\dot{P}_{\mathrm{SI}}=-\left(\gamma_{1}+\epsilon_{2}+\tau+\omega\right) P_{\mathrm{SI}}+2\left(\gamma_{1}+\epsilon_{2}\right)\left(1-P_{\mathrm{SI}}-P_{\mathrm{SS}}\right)+\tau n\left(P_{\mathrm{SS}}-\frac{P_{\mathrm{SI}}}{2}\right) \frac{P_{\mathrm{SI}}}{1-P_{I}}-\left(\beta_{1}+\varepsilon_{1}\right) P_{I} P_{\mathrm{SI}} \\
\dot{P}_{\mathrm{SS}}=\left(\gamma_{1}+\varepsilon_{2}\right) P_{\mathrm{SI}}-\tau n \frac{P_{\mathrm{SS}} P_{\mathrm{SI}}}{1-P_{I}}+\omega P_{\mathrm{SI}}+\left(\beta_{1}+\varepsilon_{1}\right) P_{I} P_{\mathrm{SI}}
\end{array}\right.
$$

where $\left(\varepsilon_{1}, \varepsilon_{2}\right)$ is in the neighborhood of $(0,0)$.

Theorem 4. If parameters $\varepsilon=\left(\varepsilon_{1}, \varepsilon_{2}\right)$ are sufficiently small and $\left(\tau, \omega, n, \gamma_{1}, \beta_{1}\right)$ satisfy the conditions in Theorem 3 , then system (44) admits a generic unfolding of the cusp singularity of codimension 2.

Proof. We present only the main steps to illustrate the proof and omit the details of calculation because they are similar to those in $[19,26]$. Shift $\widehat{E}$ to the origin by substituting
$x=P_{I}-\widehat{P}_{I}, y=P_{\mathrm{SI}}-\widehat{P}_{\mathrm{SI}}, z=P_{\mathrm{SS}}-\widehat{P}_{\mathrm{SS}}$. Then, using the method in Appendix B, we get

$$
n=\frac{(-3 \omega+3 \tau) \widehat{P}_{I}^{4}+6 \omega \widehat{P}_{I}^{3}-(\omega+5 \tau) \widehat{P}_{I}^{2}+(4 \tau-4 \omega) \widehat{P}_{I}+2 \omega-\tau}{\tau\left(2 \widehat{P}_{I}-1\right)\left(3 \widehat{P}_{I}^{2}-2\right)}
$$

Next, we apply transformation (30) to system (38) to obtain 


$$
\left\{\begin{array}{l}
\dot{\mu}=\nu+l_{00010} \varepsilon_{1}+l_{00001} \varepsilon_{2}+L_{20} \mu^{2}+L_{11} \mu \nu+L_{02} \nu^{2}+\sum_{\substack{i+j+k+p+q \geq 2 \\
i+j \neq 2 i f i+j+k+p+q=2}} l_{i j k p q} \mu^{i} \nu^{j} \xi^{k} \varepsilon_{1}^{p} \varepsilon_{2}^{q}, \\
\dot{\nu}=m_{00010} \varepsilon_{1}+m_{00001} \varepsilon_{2}+M_{20} \mu^{2}+M_{11} \mu \nu+M_{02} \nu^{2}+\sum_{\substack{i+j+k+p+q \geq 2 \\
i+j \neq 2 i f i+j+k+p+q=2}} m_{i j k p q} \mu^{i} \nu^{j} \xi^{k} \varepsilon_{1}^{p} \varepsilon_{2}^{q}, \\
\dot{\xi}=-G_{2}\left(\widehat{P}_{I}\right) \xi+g_{00010} \varepsilon_{1}+g_{00001} \varepsilon_{2}+\sum_{i+j+k+p+q \geq 2} g_{i j k p q} \mu^{i} \nu^{j} \xi^{k} \varepsilon_{1}^{p} \varepsilon_{2}^{q} .
\end{array}\right.
$$

According to the method in $[19,26]$, the local center manifold has the following form:

$$
\xi=\frac{g_{00010} \varepsilon_{1}+g_{00001} \varepsilon_{2}}{G_{2}\left(\widehat{P}_{I}\right)}+\sum_{i+j+p+q \geq 2} h_{i j p q} \mu^{i} v^{j} \varepsilon_{1}^{p} \varepsilon_{2}^{q}
$$

$$
\left\{\begin{array}{l}
\dot{\mu}=\nu+l_{00010} \varepsilon_{1}+l_{00001} \varepsilon_{2}+L_{20} \mu^{2}+L_{11} \mu \nu+L_{02} \nu^{2}+\sum_{\substack{i+j+p+q \geq 2 \\
i+j \neq 2 i f i+j+p+q=2 \\
i f j}} l_{i j p q} \mu^{i} \nu^{j} \varepsilon_{1}^{p} \varepsilon_{2}^{q}, \\
\dot{\nu}=m_{00010} \varepsilon_{1}+m_{00001} \varepsilon_{2}+M_{20} \mu^{2}+M_{11} \mu \nu+M_{02} \nu^{2}+\sum_{\substack{i+j+p+q \geq 2 \\
i+j \neq 2 i f i+j+p+q=2}} m_{i j p q} \mu^{i} \nu^{j} \varepsilon_{1}^{p} \varepsilon_{2}^{q} .
\end{array}\right.
$$

We use the near-identity transformation

$$
\begin{aligned}
\mu= & \left(1+L_{1010} \varepsilon_{1}+L_{1001} \varepsilon_{2}+\mathcal{O}\left(\varepsilon^{2}\right)\right) X+L_{0010} \varepsilon_{1}+L_{0001} \varepsilon_{2}+\mathcal{O}\left(\varepsilon^{2}\right)+\left(\mathcal{O}\left(\varepsilon^{2}\right)\right) Y \\
& +\frac{1}{2}\left(L_{11}+M_{02}+\mathcal{O}(\varepsilon)\right) X^{2}+\left(L_{02}+\mathcal{O}(\varepsilon)\right) X Y+(\mathcal{O}(\varepsilon)) Y^{2}+\mathcal{O}\left(|X, Y|^{3}\right), \\
v= & \left(1+M_{0110} \varepsilon_{1}+M_{0101} \varepsilon_{2}+\mathcal{O}\left(\varepsilon^{2}\right)\right) Y-\left(l_{00010} \varepsilon_{1}+l_{00001} \varepsilon_{2}+\mathcal{O}\left(\varepsilon^{2}\right)\right) \\
& +\left(M_{1010} \varepsilon_{1}+M_{1001} \varepsilon_{2}+\mathcal{O}\left(\varepsilon^{2}\right)\right) X-\left(L_{20}+\mathcal{O}(\varepsilon)\right) X^{2}+\left(M_{02}+\mathcal{O}(\varepsilon)\right) X Y \\
& +(\mathcal{O}(\varepsilon)) Y^{2}+\mathcal{O}\left(|X, Y|^{3}\right),
\end{aligned}
$$

and rewrite $X, Y$ into $\mu$ and $\nu$ to get

$$
\left\{\begin{array}{l}
\dot{\mu}=\nu \\
\dot{\nu}=\bar{v}_{1}(\varepsilon)+\bar{v}_{2}(\varepsilon) \nu+\left(M_{20}+\mathcal{O}\left(\varepsilon^{2}\right)\right) \mu^{2}+\left(2 L_{20}+M_{11}+\mathcal{O}(\varepsilon)\right) \mu \nu+\nu \mathcal{O}\left(|\mu, \nu|^{2}\right) .
\end{array}\right.
$$




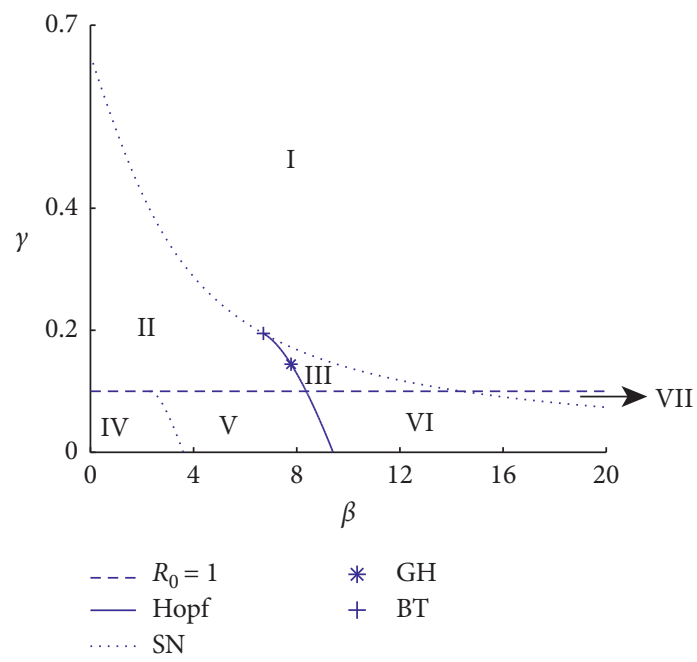

Figure 2: Bifurcation diagrams in the plane $(\beta, \gamma)$. The parameter values are fixed as $\tau=0.5, n=6, \omega=2.9$.

Now, we can rescale the time and variables by formula (35) and

$$
\left(\bar{v}_{1}(\varepsilon), \bar{v}_{2}(\varepsilon)\right) \longrightarrow\left(\frac{\left(M_{20}+\mathcal{O}\left(\varepsilon^{2}\right)\right)^{3}}{\left(2 L_{20}+M_{11}+\mathcal{O}(\varepsilon)\right)^{4}} v_{1}(\varepsilon), \frac{M_{20}+\mathcal{O}\left(\varepsilon^{2}\right)}{2 L_{20}+M_{11}+\mathcal{O}(\varepsilon)} v_{2}(\varepsilon)\right)
$$

such that system (44) becomes

$$
\left\{\begin{array}{l}
\dot{\mu}=v, \\
\dot{\nu}=v_{1}(\varepsilon)+v_{2}(\varepsilon) \nu+\mu^{2}+\mu \nu+\nu \mathcal{O}\left(|\mu, \nu|^{2}\right) .
\end{array}\right.
$$

Here, we omit the $v(\varepsilon)$ expansions because of the complexity and only verify the nondegeneracy conditions. After a series of calculations, we get

$$
\left|\frac{\partial\left(v_{1}, v_{2}\right)}{\partial\left(\varepsilon_{1}, \varepsilon_{2}\right)}\right|_{\varepsilon=0}=-\frac{4\left(3 \widehat{P}_{I}^{2}-2\right)^{2}\left(2 \widehat{P}_{I}-1\right)^{5} A B^{5}}{\tau^{3} \widehat{P}_{I}^{3}\left(2 \widehat{P}_{I}^{2}-2 \widehat{P}_{I}+1\right)^{8} C^{5}},
$$

where

$$
\begin{aligned}
A= & 3(\omega-\tau) \widehat{P}_{I}^{6}+15(\tau-\omega) \widehat{P}_{I}^{5}+(10 \omega-13 \tau) \widehat{P}_{I}^{4} \\
& +(10 \omega+4 \tau) \widehat{P}_{I}^{3}-(8 \omega+9 \tau) \widehat{P}_{I}^{2}+9 \tau \widehat{P}_{I}-2 \tau, \\
B= & 3(\omega-\tau) \widehat{P}_{I}^{3}+6 \tau \widehat{P}_{I}^{2}-(2 \omega+5 \tau) \widehat{P}_{I}+2 \tau, \\
C= & 3(\omega-\tau) \widehat{P}_{I}^{3}-3(\omega-\tau) \widehat{P}_{I}^{2}-(2 \omega+\tau) \widehat{P}_{I}+2 \omega .
\end{aligned}
$$

Since $\sqrt{6} / 3<\widehat{P}_{I}<1$, it is sufficient to prove that $A B C \neq 0$. If $A=0$, we get

$$
\omega=\frac{\tau\left(3 \widehat{P}_{I}^{6}-15 \widehat{P}_{I}^{5}+13 \widehat{P}_{I}^{4}-4 \widehat{P}_{I}^{3}+9 \widehat{P}_{I}^{2}-9 \widehat{P}_{I}+2\right)}{\widehat{P}_{I}^{2}\left(\widehat{P}_{I}-1\right)\left(\widehat{P}_{I}-4\right)\left(3 \widehat{P}_{I}^{2}-2\right)}<0,
$$

which leads to contradiction. Similarly, it can be proved that $B C \neq 0$. Therefore, the nondegeneracy property holds, and the proof is completed.

\section{Numerical Simulations}

In this section, we use numerical software MATCONT to demonstrate how the dynamical behaviors of model (5) are influenced by the parameters [31-33]. First, we choose $\beta$ and $\gamma$ as bifurcation parameters to obtain the bifurcation diagrams in Figure 2. The plane is split into seven regions by the saddle-node bifurcation curve, the Hopf bifurcation curve, and the line of $R_{0}=1$. The distribution of endemic equilibria and their stability are described in Table 2. Furthermore, the Hopf bifurcation curve meets the saddle-node bifurcation curve at a Bogdanov-Takens point (BT) and is divided into two parts by the degenerate Hopf bifurcation point $(\mathrm{GH})$, where the upper part is subcritical Hopf and the lower part is supercritical.

It is notable that the Bogdanov-Takens point implies the existence of a homoclinic orbit. Thus, one can select the parameters in region II in Figure 2 such that a homoclinic orbit coexists with a stable endemic equilibrium. This is the case if $\beta=7.89$ with $\tau=0.5, n=6, \omega=2.9, \gamma=0.13$, as shown in Figure 3(a).

Interestingly, an unstable periodical solution emerges as $\beta$ increases due to the homoclinic orbit is broken, which is shown in Figure 3(b), where $\beta=7.91$. As $\beta$ increases further, the equilibrium $E_{1}$ becomes unstable, and a stable periodic solution appears due to the supercritical Hopf bifurcation 
TABLE 2: Stability of the steady states in different regions in Figure 2.

\begin{tabular}{lcccc}
\hline & DEF & $E_{1}$ & $E_{2}$ & $E_{3}$ \\
\hline I & Stable & Does not exist & Does not exist & Does not exist \\
II & Stable & Stable & Unstable & Does not exist \\
III & Stable & Unstable & Unstable & Does not exist \\
IV & Unstable & Stable & Does not exist & Does not exist \\
V & Unstable & Unstable & Stable \\
VI & Unstable & Unstable & Unstable & Stable \\
VII & Unstable & Does not exist & Does not exist & Stable \\
\hline
\end{tabular}

$E_{1}, E_{2}$, and $E_{3}$ are three endemic steady states arranged according to sizes.

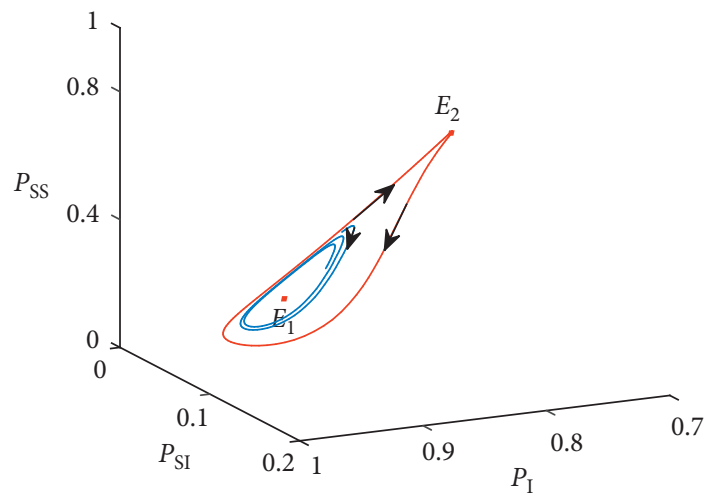

(a)

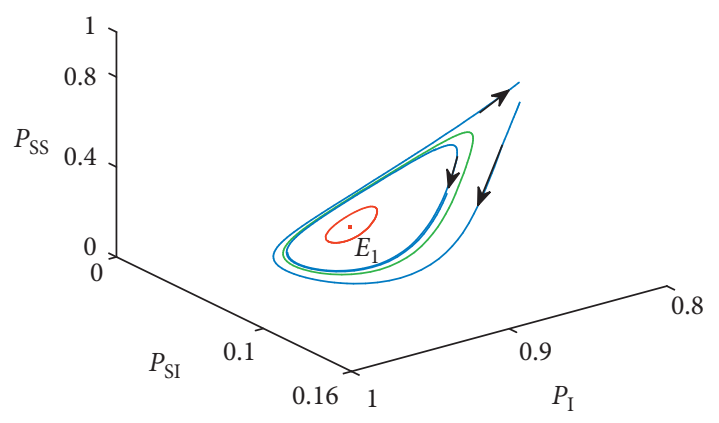

(c)

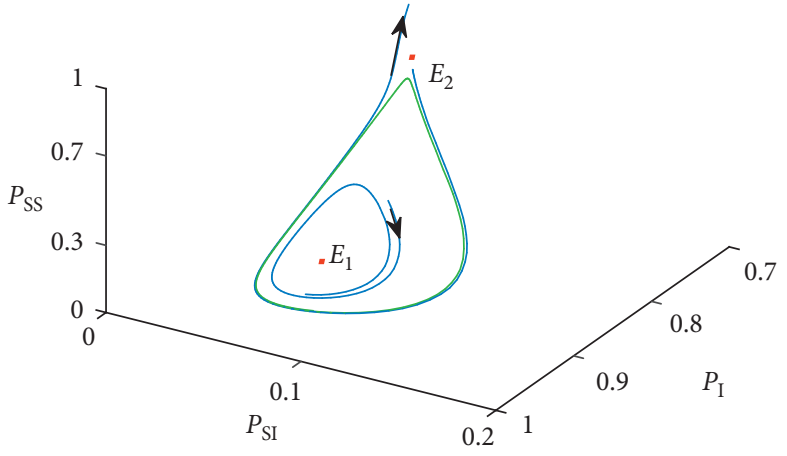

(b)

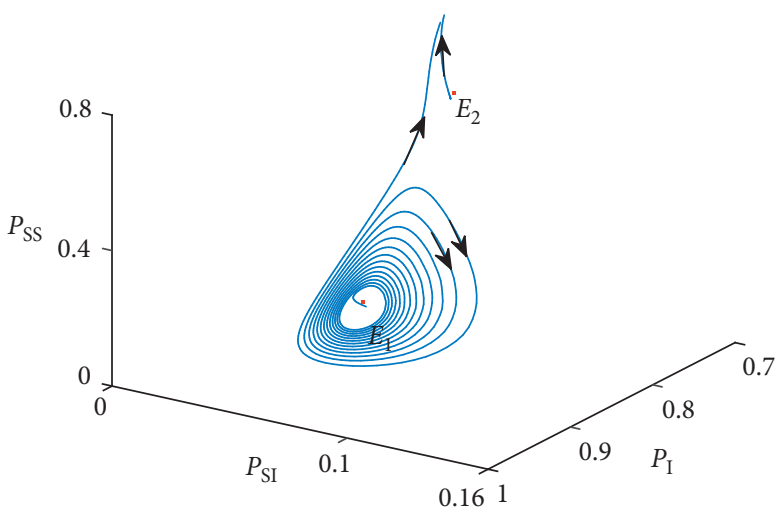

(d)

FIgURe 3: Global dynamics of system (5), where $\tau=0.5, n=6, \omega=2.9$, and $\gamma=0.13$. (a) Coexistence of a homoclinic orbit with a stable equilibrium in region II, where $\beta=7.89$. (b) Coexistence of an unstable cycle with a stable equilibrium in region II, where $\beta=7.91$. (c) Coexistence of an unstable cycle with a stable cycle in region III, where $\beta=7.99$. (d) $E_{1}$ and $E_{2}$ are unstable, and orbits tend to the diseasefree equilibrium in region III, where $\beta=8.1$.

when $\beta=7.99$ (see Figure 3(c)). Thus, the system has two limit cycles. The unstable limit cycle shrinks, and the stable limit cycle expands as the rise of $\beta$ until these two cycles coalesce with each other at $\beta=8.01$. Finally, system (5) has no periodic solution, and its orbits except for the endemic equilibria with the stable manifolds of the saddle tend to the disease-free equilibrium when $\beta>8.01$, as shown in Figure 3(d).
In order to show the influence of rewiring coefficient $\beta$ on $P_{I}$, we choose $\beta$ as a bifurcation parameter to obtain the bifurcation diagrams in Figure 4(a). As $\beta$ increases gradually from 0 , system (5) has only one equilibrium which is globally asymptotically stable. Therefore, the final size of $P_{I}$ settles down to the higher state. As $\beta$ passes through $\beta_{1}$ and stays in $\left(\beta_{1}, \beta_{h}\right)$, the final size of $P_{I}$ depends on the initial size of $P_{I}$. If the initial size of $P_{I}$ is smaller, the final size of $P_{I}$ stays in the 


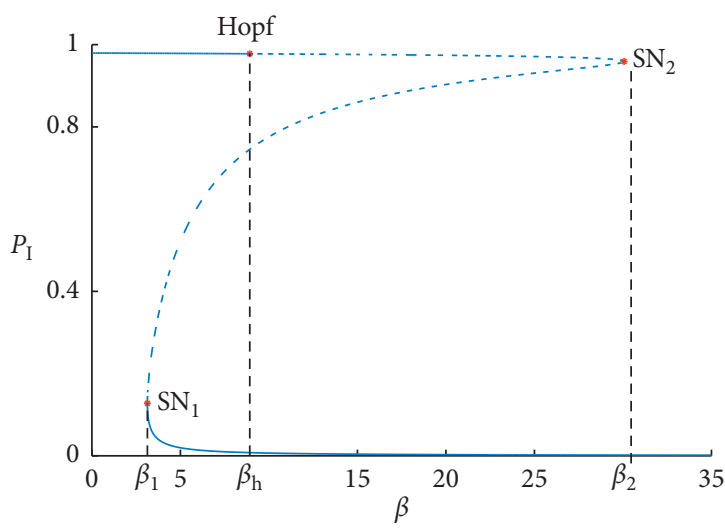

(a)

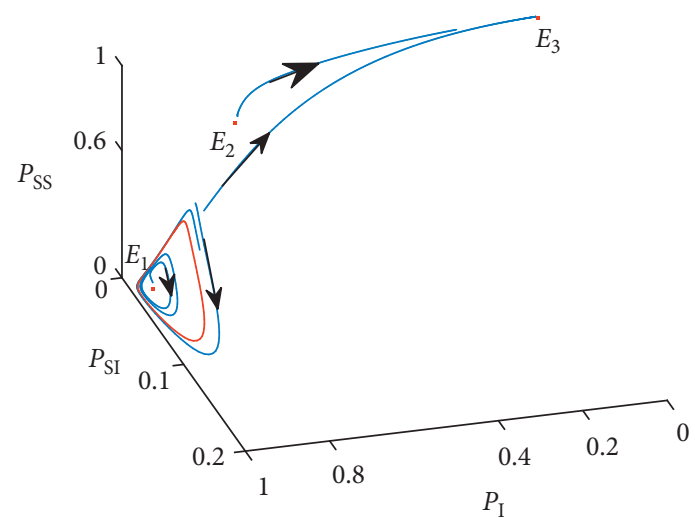

(b)

FiguRe 4: (a) Bifurcation diagram of equilibria. $\mathrm{SN}_{1}$ and $\mathrm{SN}_{2}$ represent saddle-node bifurcations, Hopf stands for a Hopf bifurcation, and solid (dotted) curves represent stable (unstable) equilibrium states. The parameter values are $\tau=0.5, n=6, \omega=2.9$, and $\gamma=0.05$. (b) Coexistence of a stable cycle with a stable equilibrium. A stable cycle is shown by the red curve, and a stable equilibrium is represented by $E_{3}$, where $\beta=9.60357$ is close to $\beta_{h}$ shown in (a).

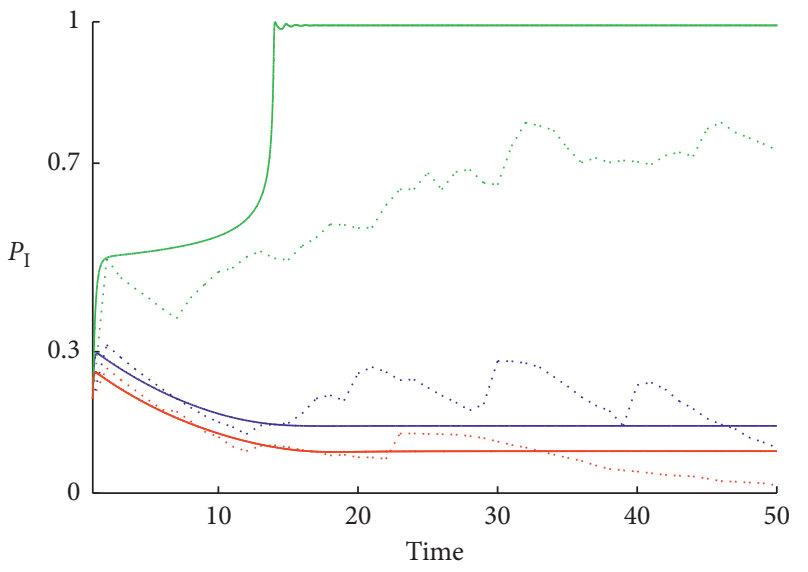

Figure 5: Comparison of the time evolution of prevalence in pairwise model (5) and simulation models, with the continuous curves which are obtained from system (5), and the dotted curves are obtained from average of 50 simulations. The parameter values are $\gamma=0.1, \tau=$ $1.5, n=10, \omega=2.9$, and $\beta=0.05$ (green), 0.1 (blue), or 0.15 (red). The initial value is $P_{I}=0.2, P_{\mathrm{SI}}=0.19$, and $P_{\mathrm{SS}}=0.76$.

lower branch of the bifurcation curve. Otherwise, the final size approaches the upper stable branch. When $\beta$ crosses $\beta_{h}$, the lager equilibrium point loses stability due to the occurrence of supercritical Hopf bifurcation, and a stable periodical solution emerges. As $\beta>\beta_{2}$, the system has only one equilibrium which is globally stable and has very low value. It is clear that the rewiring coefficient $\beta$ tends to decrease the endemic level.

Finally, we compare model (5) with stochastic simulations. The random network is generated by using the Gillespie algorithm [34]. In stochastic simulations, the initial network degree distribution of each sample is basically the same, which obeys the Poisson distribution, but the specific structure is different. It is shown in Figure 5 that model (5) and the simulations fit well when $\beta$ is suitably large to cause a low infection rate. However, when $\beta$ is smaller than a critical value, the error of model (5) to stochastic simulations increases, although the trend of value $P_{I}$ in the model and simulation are the same. This is because the strong adaptive response from the higher coefficient $\beta$ decreases the infection to low level so that the degree distribution of the network is approximated well by the Poisson distribution. Therefore, approximation (3) is very suitable for the moment closure.

\section{Discussion}

In this paper, we have extended the network model in [16] by incorporating the adaptive rewiring rate, that is, the constant 
rewiring rate of the model in [16] is allowed to be the rate which is correlated with the infective number to describe the self-protection of individuals to reduce infection risk. Through rigorous mathematical analysis, we have obtained the conditions for the backward bifurcation, Hopf bifurcation, and Bogdanov-Takens bifurcation. These extend the mathematical analysis in [19]. Interestingly, the coefficient $\beta$ of adaptive rewiring can induce the complicated behaviors of model (5). As shown in Figure 1, the model exhibits the bifurcation curve with " $\mathrm{S}$ " shape due to the adaptive rewiring rate, which leads to multiple equilibria and twice saddlenode bifurcations. More importantly, the disease can persist even though the basic reproduction number is less than unity when the bifurcation at the disease-free equilibrium is forward. Moreover, we find a scenario where a stable periodic solution coexists with a stable equilibrium, which is shown in Figure 4(b). Finally, by Figure 4(a), we see that increasing $\beta$ tends to decrease the infection level, although $\beta$ has no direct contribution to $R_{0}$ from Section 2 .
From the previous theoretical analysis and simulations, we can infer that if the magnitude of rewiring is positively related to the scale of infection, it produces remarkable help for the epidemic control. Especially if the adaptive response is strong enough, the epidemic outbreak can be contained.

We have investigated the qualitative behaviors of system (5). It is very interesting to apply the theoretical results to the problems with real data, as done in the paper [35]. It would also be important to consider the case where the rewiring rate is a saturated function of infected nodes and consider the time delay of adaptive rewiring. We leave these as future projects.

\section{Appendix}

\section{A. Transcritical Bifurcation}

Let $X_{1}=P_{I}, X_{2}=P_{\mathrm{SI}}$, and $X_{3}=1-P_{\mathrm{SS}}$. Then, system (5) can be rewritten as

$$
\left\{\begin{array}{l}
\dot{X}_{1}=\frac{\tau n}{2} X_{2}-\gamma X_{1} \\
\dot{X}_{2}=-\gamma X_{2}+2 \gamma\left(X_{3}-X_{2}\right)-\tau X_{2}-\frac{\tau n}{2} \frac{X_{2} X_{2}}{1-X_{1}}+\tau n \frac{\left(1-X_{3}\right) X_{2}}{1-X_{1}}-\omega X_{2}-\beta X_{1} X_{2} \\
\dot{X}_{3}=-\gamma X_{2}+\tau n \frac{\left(1-X_{3}\right) X_{2}}{1-X_{1}}-\omega X_{2}-\beta X_{1} X_{2}
\end{array}\right.
$$

Let $\phi=\tau-\tau_{c}$ and $\tau_{c}=(\gamma+\omega) / n$ with $\tau=(\gamma+\omega) / n$. Then, the Jacobian matrix at $(0,0,0)$ is given by

$$
J(0,0,0)=\left(\begin{array}{ccc}
-\gamma & \frac{\gamma+\omega}{2} & 0 \\
0 & -2 \gamma-\frac{\gamma+\omega}{n} & 2 \gamma \\
0 & 0 & 0
\end{array}\right)
$$

Obviously, 0 is a simple eigenvalue of $J(0,0,0)$. A right eigenvector associated to the zero eigenvalue is $v=((\gamma+\omega / 2) n, \gamma n,(2 \gamma n+\gamma+\omega / 2))^{\prime}$, and the left eigenvector $\nu$ which satisfies $\nu \cdot v=1$ is $\nu=(0,0,(2 / 2 \gamma n+\gamma+\omega))$.

We write system (A.1) as

$$
\dot{X}(t)=F(X(t), \phi) .
$$

By the computation formulae in $[14,27]$, we get

$$
\begin{aligned}
a_{0} & :=\sum_{k, i, j=1}^{3} v_{k} v_{i} v_{j} \frac{\partial^{2} F_{k}}{\partial X_{i} \partial X_{j}}(0,0,0,0) \\
& =\sum_{i, j=1}^{3} v_{3} v_{i} v_{j} \frac{\partial^{2} F_{3}}{\partial X_{i} \partial X_{j}}(0,0,0,0) \\
& =2(\gamma+\omega) \gamma n\left(\frac{-\beta+\gamma+\omega}{(2 n+1) \gamma+\omega} n-1\right), \\
b_{0} & :=\sum_{k, i=1}^{3} v_{k} v_{i} \frac{\partial^{2} F_{k}}{\partial X_{i} \partial \phi}(0,0,0,0) \\
& =\sum_{i=1}^{3} v_{3} v_{i} \frac{\partial^{2} F_{3}}{\partial X_{i} \partial \phi}(0,0,0,0) \\
& =\frac{2 \gamma n^{2}}{2 \gamma n+\gamma+\omega} .
\end{aligned}
$$

Consequently, from $[14,27]$, we conclude the results of Theorem 1.

\section{B. Supplementary to the Proof of Theorem 3}

The coefficients in (31) are-where 


$$
\begin{aligned}
& L_{20}=\frac{-n \tau \gamma \widetilde{L}_{20}}{\left(2 \beta \widehat{P}_{I}-\beta+\gamma+\omega\right)^{2}\left(\widehat{P}_{I}-1\right)^{3}\left(\gamma \widehat{P}_{I}-\tau \widehat{P}_{I}+3 \gamma+\tau\right)^{2}}, \\
& L_{11}=\frac{n \tau \widetilde{L}_{11}}{2 \widehat{P}_{I}\left(2 \beta \widehat{P}_{I}-\beta+\gamma+\omega\right)^{2}\left(\widehat{P}_{I}-1\right)^{3}\left(\gamma \widehat{P}_{I}-\tau \widehat{P}_{I}+3 \gamma+\tau\right)^{2}}, \\
& L_{02}=\frac{-n \tau \widetilde{L}_{02}}{2 \gamma\left(2 \beta \widehat{P}_{I}-\beta+\gamma+\omega\right)^{2}\left(\widehat{P}_{I}-1\right)^{3}\left(\gamma \widehat{P}_{I}-\tau \widehat{P}_{I}+3 \gamma+\tau\right)^{2}}, \\
& M_{20}=\frac{-2 n \tau \widehat{P}_{I} \gamma^{2} \widetilde{M}_{20}}{\left(2 \beta \widehat{P}_{I}-\beta+\gamma+\omega\right)^{2}\left(\widehat{P}_{I}-1\right)^{3}\left(\gamma \widehat{P}_{I}-\tau \widehat{P}_{I}+3 \gamma+\tau\right)}, \\
& M_{11}=\frac{n \tau \gamma \tilde{M}_{11}}{\left(2 \beta \widehat{P}_{I}-\beta+\gamma+\omega\right)^{2}\left(\widehat{P}_{I}-1\right)^{3}\left(\gamma \widehat{P}_{I}-\tau \widehat{P}_{I}+3 \gamma+\tau\right)}, \\
& M_{02}=\frac{-n \tau \widehat{P}_{I} \tilde{M}_{02}}{\left(2 \beta \widehat{P}_{I}-\beta+\gamma+\omega\right)^{2}\left(\widehat{P}_{I}-1\right)^{3}\left(\gamma \widehat{P}_{I}-\tau \widehat{P}_{I}+3 \gamma+\tau\right)}, \\
& \widetilde{L}_{20}=\left(-6 \beta^{2} \gamma+2 \beta^{2} \tau\right) \widehat{P}_{I}^{6}+\left(11 \beta^{2} \gamma-5 \beta^{2} \tau+5 \beta \gamma^{2}-3 \beta \gamma \omega+5 \beta \gamma \tau+\beta \omega \tau-4 \beta \tau^{2}\right) \widehat{P}_{I}^{5} \\
& +\left(-8 \beta^{2} \gamma+2 \beta^{2} \tau-20 \beta \gamma^{2}+4 \beta \gamma \omega+10 \beta \gamma \tau-2 \beta \omega \tau+12 \beta \tau^{2}\right) \widehat{P}_{I}^{4} \\
& +\left(10 \beta^{2} \gamma+4 \beta^{2} \tau-4 \beta \gamma^{2}-2 \beta \gamma \omega-38 \beta \gamma \tau-12 \beta \tau^{2}\right) \widehat{P}_{I}^{3} \\
& +\left(-10 \beta^{2} \gamma-4 \beta^{2} \tau+29 \beta \gamma^{2}+4 \beta \gamma \omega+23 \beta \gamma \tau+2 \beta \omega \tau+4 \beta \tau^{2}+3 \gamma^{3}+3 \gamma^{2} \omega-\gamma^{2} \tau-\gamma \omega \tau\right) \widehat{P}_{I}^{2} \\
& +\left(3 \beta^{2} \gamma+\beta^{2} \tau+\beta \gamma^{2}-3 \beta \gamma \omega+\beta \gamma \tau-\beta \omega \tau+2 \gamma^{3}+2 \gamma^{2} \omega\right) \widehat{P}_{I} \\
& -3 \beta \gamma^{2}-\beta \gamma \tau+3 \gamma^{3}+3 \gamma^{2} \omega+\gamma^{2} \tau+\gamma \omega \tau, \\
& \widetilde{L}_{11}=\left(-24 \beta^{2} \gamma+8 \beta^{2} \tau\right) \widehat{P}_{I}^{7} \\
& +\left(56 \beta^{2} \gamma-24 \beta^{2} \tau+20 \beta \gamma^{2}-12 \beta \gamma \omega+20 \beta \gamma \tau+4 \beta \omega \tau-16 \tau^{2} \beta\right) \widehat{P}_{I}^{6} \\
& +\left(-60 \beta^{2} \gamma+20 \beta^{2} \tau-60 \beta \gamma^{2}+28 \beta \gamma \omega+20 \beta \gamma \tau-12 \beta \omega \tau+56 \tau^{2} \beta\right) \widehat{P}_{I}^{5} \\
& +\left(67 \beta^{2} \gamma+7 \beta^{2} \tau-2 \beta \gamma^{2}-30 \beta \gamma \omega-150 \beta \gamma \tau+10 \beta \omega \tau-76 \tau^{2} \beta+7 \gamma^{3}+10 \gamma^{2} \omega-9 \gamma^{2} \tau\right. \\
& \left.+3 \gamma \omega^{2}-10 \gamma \omega \tau+4 \gamma \tau^{2}-\omega^{2} \tau+4 \tau^{2} \omega\right) \widehat{P}_{I}^{4} \\
& +\left(-68 \beta^{2} \gamma-22 \beta^{2} \tau+136 \beta \gamma^{2}+32 \beta \gamma \omega+176 \beta \gamma \tau+4 \beta \omega \tau\right. \\
& \left.+52 \tau^{2} \beta+28 \gamma^{3}+24 \gamma^{2} \omega-10 \gamma^{2} \tau-4 \gamma \omega^{2}-8 \gamma \omega \tau-12 \gamma \tau^{2}+2 \omega^{2} \tau-12 \tau^{2} \omega\right) \widehat{P}_{I}^{3} \\
& +\left(42 \beta^{2} \gamma+16 \beta^{2} \tau-100 \beta \gamma^{2}-32 \beta \gamma \omega-92 \beta \gamma \tau\right. \\
& \left.-12 \beta \omega \tau-20 \tau^{2} \beta+22 \gamma^{3}+24 \gamma^{2} \omega+40 \gamma^{2} \tau+2 \gamma \omega^{2}+40 \gamma \omega \tau+12 \gamma \tau^{2}+12 \tau^{2} \omega\right) \widehat{P}_{I}^{2} \\
& +\left(-16 \beta^{2} \gamma-6 \beta^{2} \tau+44 \beta \gamma^{2}+20 \beta \gamma \omega+28 \beta \gamma \tau+8 \beta \omega \tau+4 \tau^{2} \beta-28 \gamma^{3}-32 \gamma^{2} \omega\right. \\
& \left.-22 \gamma^{2} \tau-4 \gamma \omega^{2}-24 \gamma \omega \tau-4 \gamma \tau^{2}-2 \omega^{2} \tau-4 \tau^{2} \omega\right) \widehat{P}_{I} \\
& +3 \beta^{2} \gamma+\beta^{2} \tau-6 \beta \gamma^{2}-6 \beta \gamma \omega-2 \beta \gamma \tau-2 \beta \omega \tau+3 \gamma^{3}+6 \gamma^{2} \omega+\gamma^{2} \tau+3 \gamma \omega^{2}+2 \gamma \omega \tau+\omega^{2} \tau \text {, }
\end{aligned}
$$




$$
\begin{aligned}
& \widetilde{L}_{02}=\left(-6 \beta^{2} \gamma+2 \beta^{2} \tau+18 \beta \gamma^{2}+6 \beta \gamma \omega-6 \beta \gamma \tau-2 \beta \omega \tau\right) \widehat{P}_{I}^{5} \\
& +\left(11 \beta^{2} \gamma-5 \beta^{2} \tau-4 \beta \gamma^{2}-14 \beta \gamma \omega+12 \beta \gamma \tau+6 \beta \omega \tau-4 \beta \tau^{2}+\gamma^{3}+4 \gamma^{2} \omega-7 \gamma^{2} \tau\right. \\
& \left.+3 \gamma \omega^{2}-8 \gamma \omega \tau+4 \gamma \tau^{2}-\omega^{2} \tau+4 \omega \tau^{2}\right) \widehat{P}_{I}^{4} \\
& +\left(-8 \beta^{2} \gamma+2 \beta^{2} \tau-4 \beta \gamma^{2}+12 \beta \gamma \omega+12 \beta \gamma \tau-4 \beta \omega \tau+12 \beta \tau^{2}+24 \gamma^{3}+20 \gamma^{2} \omega\right. \\
& \left.-10 \gamma^{2} \tau-4 \gamma \omega^{2}-8 \gamma \omega \tau-12 \gamma \tau^{2}+2 \omega^{2} \tau-12 \omega \tau^{2}\right) \widehat{P}_{I}^{3} \\
& +\left(10 \beta^{2} \gamma+4 \beta^{2} \tau-26 \beta \gamma^{2}-12 \beta \gamma \omega-42 \beta \gamma \tau-4 \beta \omega \tau-12 \beta \tau^{2}+16 \gamma^{3}+18 \gamma^{2} \omega\right. \\
& \left.+38 \gamma^{2} \tau+2 \gamma \omega^{2}+38 \gamma \omega \tau+12 \gamma \tau^{2}+12 \omega \tau^{2}\right) \widehat{P}_{I}^{2} \\
& +\left(-10 \beta^{2} \gamma-4 \beta^{2} \tau+38 \beta \gamma^{2}+14 \beta \gamma \omega+26 \beta \gamma \tau+6 \beta \omega \tau+4 \beta \tau^{2}-28 \gamma^{3}-32 \gamma^{2} \omega\right. \\
& \left.-22 \gamma^{2} \tau-4 \gamma \omega^{2}-24 \gamma \omega \tau-4 \gamma \tau^{2}-2 \omega^{2} \tau-4 \omega \tau^{2}\right) \widehat{P}_{I} \\
& +3 \beta^{2} \gamma+\beta^{2} \tau-6 \beta \gamma^{2}-6 \beta \gamma \omega-2 \beta \gamma \tau-2 \beta \omega \tau+3 \gamma^{3}+6 \gamma^{2} \omega+\gamma^{2} \tau+3 \gamma \omega^{2}+2 \gamma \omega \tau+\omega^{2} \tau \text {, } \\
& \tilde{M}_{20}=-2 \beta^{2} \widehat{P}_{I}^{4}+\left(5 \beta^{2}+\beta \gamma-\beta \omega+2 \beta \tau\right) \widehat{P}_{I}^{3}+\left(-4 \beta^{2}-6 \beta \gamma+2 \beta \omega-4 \beta \tau\right) \widehat{P}_{I}^{2}+\left(\beta^{2}+7 \beta \gamma-\beta \omega+2 \beta \tau\right) \widehat{P}_{I} \\
& -\beta \gamma+\gamma^{2}+\gamma \omega \\
& \tilde{M}_{11}=-8 \beta^{2} \widehat{P}_{I}^{5}+\left(24 \beta^{2}+4 \beta \gamma-4 \beta \omega+8 \beta \tau\right) \widehat{P}_{I}^{4}+\left(-28 \beta^{2}-16 \beta \gamma+12 \beta \omega-20 \beta \tau\right) \widehat{P}_{I}^{3} \\
& +\left(17 \beta^{2}+24 \beta \gamma-14 \beta \omega+18 \beta \tau+3 \gamma^{2}+4 \gamma \omega-2 \gamma \tau+\omega^{2}-2 \omega \tau\right) \widehat{P}_{I}^{2} \\
& +\left(-6 \beta^{2}-12 \beta \gamma+8 \beta \omega-8 \beta \tau+6 \gamma^{2}+4 \gamma \omega+4 \gamma \tau-2 \omega^{2}+4 \omega \tau\right) \widehat{P}_{I} \\
& +\beta^{2}+4 \beta \gamma-2 \beta \omega+2 \beta \tau-5 \gamma^{2}-4 \gamma \omega-2 \gamma \tau+\omega^{2}-2 \omega \tau, \\
& \widetilde{M}_{02}=\left(-2 \beta^{2}+6 \beta \gamma+2 \beta \omega\right) \widehat{P}_{I}^{3}+\left(5 \beta^{2}-6 \beta \gamma-6 \beta \omega+2 \beta \tau+\gamma^{2}+2 \gamma \omega-2 \gamma \tau+\omega^{2}-2 \omega \tau\right) \widehat{P}_{I}^{2} \\
& +\left(-4 \beta^{2}-2 \beta+6 \beta \omega-4 \beta \tau+6 \gamma^{2}+4 \gamma \omega+4 \gamma \tau-2 \omega^{2}+4 \omega \tau\right) \widehat{P}_{I} \\
& +\beta^{2}+4 \beta \gamma-2 \beta \omega+2 \beta \tau-5 \gamma^{2}-4 \gamma \omega-2 \gamma \tau+\omega^{2}-2 \omega \tau \text {. }
\end{aligned}
$$

Now, we are able to prove $M_{20}>0$ and $M_{11}+2 L_{20}>0$. Notice that the endemic steady state $\widehat{E}=\left(\widehat{P}_{I}, \widehat{P}_{\text {SI }}, \widehat{P}_{\text {SS }}\right)$ satisfies $F\left(\widehat{P}_{I}\right)=0, G_{0}\left(\widehat{P}_{I}\right)=0$, and $G_{1}\left(\widehat{P}_{I}\right)=0$, which can be expressed as follows:

$$
\left\{\begin{array}{l}
0=\beta \widehat{P}_{I}^{3}+(\omega-2 \beta-\tau) \widehat{P}_{I}^{2}+(-2 \omega+\beta+\tau+\tau n) \widehat{P}_{I}+(\gamma+\omega-\tau n) \\
0=2 \beta \widehat{P}_{I}^{3}+(-3 \beta+\omega-\tau) \widehat{P}_{I}^{2}+(\beta+3 \gamma-\omega) \widehat{P}_{I}+2 \gamma+\tau \\
0=2 \beta \widehat{P}_{I}^{3}+(-5 \beta+\omega-\tau) \widehat{P}_{I}^{2}+(4 \beta-2 \omega+2 \tau) \widehat{P}_{I}-\beta-\gamma+\omega-\tau .
\end{array}\right.
$$

From the equations in (B.3), we obtain

$$
\left\{\begin{array}{l}
n=\frac{\left(3 \widehat{P}_{I}^{2}-2\right)\left(\widehat{P}_{I}-1\right)^{2} \beta+\widehat{P}_{I} \tau\left(3 \widehat{P}_{I}-2\right)}{\tau\left(3 \widehat{P}_{I}^{2}-2\right)}, \\
\omega=-\frac{\left(2 \widehat{P}_{I}-1\right)\left(3 \widehat{P}_{I}^{2}-2\right) \beta-\tau\left(3 \widehat{P}_{I}^{2}-1\right)}{3 \widehat{P}_{I}^{2}-2}, \\
\gamma=\frac{\left(\widehat{P}_{I}-1\right)^{2} \tau}{3 \widehat{P}_{I}^{2}-2} .
\end{array}\right.
$$

where

$$
\begin{aligned}
& Q_{1}=\frac{\left(\left(3 \widehat{P}_{I}^{2}-2\right)\left(\widehat{P}_{I}-1\right)^{2} \beta+\widehat{P}_{I} \tau\left(3 \widehat{P}_{I}-2\right)\right)\left(\widehat{P}_{I}-1\right)^{2}}{\left(2 \widehat{P}_{I}^{2}-2 \widehat{P}_{I}+1\right)\left(2 \widehat{P}_{I}-1\right)\left(3 \widehat{P}_{I}^{2}-2\right)^{2}}, \\
& Q_{2}=\frac{\left(\left(3 \widehat{P}_{I}^{2}-2\right)\left(\widehat{P}_{I}-1\right)^{2} \beta+\widehat{P}_{I} \tau\left(3 \widehat{P}_{I}-2\right)\right)\left(1-\widehat{P}_{I}\right)}{\widehat{P}_{I} \tau\left(2 \widehat{P}_{I}^{2}-2 \widehat{P}_{I}+1\right)^{2}\left(2 \widehat{P}_{I}-1\right)\left(3 \widehat{P}_{I}^{2}-2\right)}, \\
& S_{1}=\left(\widehat{P}_{I}-1\right)\left(3 \widehat{P}_{I}^{2}-2\right) \beta+\tau, \\
& S_{2}=\widehat{P}_{I}\left(2 \widehat{P}_{I}-1\right)\left(3 \widehat{P}_{I}^{2}-2\right) \beta-2 \tau\left(3 \widehat{P}_{I}^{2}-3 \widehat{P}_{I}+1\right) .
\end{aligned}
$$


Since $\gamma>0$, we have $\widehat{P}_{I}>\sqrt{6} / 3$, and (6) implies $\widehat{P}_{I}<1$. Hence, we obtain $Q_{1}>0$ and $Q_{2}>0$. As a result, $M_{20}$ and $M_{11}+2 L_{20}$ have the same signs as those of $S_{1}$ and $-S_{2}$, respectively.

When $\beta=0$, it is obvious that $S_{1}>0$ and $-S_{2}>0$. Thus, $M_{20}>0$ and $M_{11}+2 L_{20}>0$. Next, we assume $\beta>0$. It follows from (B.4) that

$$
\tau \geq \frac{\left(2 \widehat{P}_{I}-1\right)\left(3 \widehat{P}_{I}^{2}-2\right) \beta}{3 \widehat{P}_{I}^{2}-1} .
$$

As a consequence, we use $\widehat{P}_{I}>\sqrt{6} / 3$ and $\widehat{P}_{I}<1$ to obtain

$$
\begin{aligned}
-S_{2}= & -\widehat{P}_{I}\left(2 \widehat{P}_{I}-1\right)\left(3 \widehat{P}_{I}^{2}-2\right) \beta+2 \tau\left(3 \widehat{P}_{I}^{2}-3 \widehat{P}_{I}+1\right) \\
\geq & -\widehat{P}_{I}\left(2 \widehat{P}_{I}-1\right)\left(3 \widehat{P}_{I}^{2}-2\right) \beta+2 \frac{\left(2 \widehat{P}_{I}-1\right)\left(3 \widehat{P}_{I}^{2}-2\right) \beta}{3 \widehat{P}_{I}^{2}-1} \\
& \cdot\left(3 \widehat{P}_{I}^{2}-3 \widehat{P}_{I}+1\right) \\
= & \beta \frac{-\left(\widehat{P}_{I}-1\right)\left(2 \widehat{P}_{I}-1\right)\left(3 \widehat{P}_{I}^{2}-3 \widehat{P}_{I}+2\right)\left(3 \widehat{P}_{I}^{2}-2\right)}{3 \widehat{P}_{I}^{2}-1} \\
> & 0, \\
S_{1}= & \left(\widehat{P}_{I}-1\right)\left(3 \widehat{P}_{I}^{2}-2\right) \beta+\tau \\
\geq & \left(\widehat{P}_{I}-1\right)\left(3 \widehat{P}_{I}^{2}-2\right) \beta+\frac{\left(2 \widehat{P}_{I}-1\right)\left(3 \widehat{P}_{I}^{2}-2\right) \beta}{3 \widehat{P}_{I}^{2}-1} \\
= & \beta\left(3 \widehat{P}_{I}^{2}-2\right) \frac{\widehat{P}_{I}\left(3 \widehat{P}_{I}^{2}-3 \widehat{P}_{I}+1\right)}{3 \widehat{P}_{I}^{2}-1}
\end{aligned}
$$$$
>0 \text {. }
$$

This verifies $M_{20}>0$ and $M_{11}+2 L_{20}>0$.

\section{Data Availability}

The data used to support the findings of this study are available from the corresponding author upon request.

\section{Conflicts of Interest}

The authors declare that no conflicts of interest exist with regard to the publication of this paper.

\section{Acknowledgments}

This research was supported by the National Natural Science Foundation of China (no. 11571284).

\section{References}

[1] M. J. Keeling and K. T. D. Eames, "Networks and epidemic models," Journal of the Royal Society Interface, vol. 2, no. 4, pp. 295-307, 2005.

[2] M. Kuperman and G. Abramson, "Small world effect in an epidemiological model," Physical Review Letters, vol. 86, no. 13, pp. 2909-2912, 2001.

[3] M. E. J. Newman, "Spread of epidemic disease on networks," Physical Review E, vol. 66, no. 1, Article ID 016128, 2002.

[4] D. Normile, "The metropole, superspreaders and other mysteries," Science, vol. 339, no. 6125, pp. 1272-1273, 2013.

[5] J. Zhang, W. Jing, W. Zhang, and Z. Jin, "Avian influenza A (H7N9) model based on poultry transport network in China," Computational and Mathematical Methods in Medicine, vol. 2018, no. 4, 14 pages, 2018.

[6] X. Pei, Z. Jin, W. Zhang, and Y. Wang, "Detection of infection sources for avian influenza A (H7N9) in live poultry transport network during the fifth wave in China," IEEE Access, vol. 7, pp. 155759-155778, 2019.

[7] P. Wang, M. C. Gonzalez, C. A. Hidalgo, and A.-L. Barabasi, "Understanding the spreading patterns of mobile phone viruses," Science, vol. 324, no. 5930, pp. 1071-1076, 2009.

[8] G. Meng, M. Patrick, Y. Xue, Y. Liu, and J. Zhang, "Securing android app markets via modeling and predicting malware spread between markets," IEEE Transactions on Information Forensics and Security, vol. 14, no. 7, pp. 1944-1959, 2019.

[9] T. Brett, G. Loukas, Y. Moreno, and N. Perra, "Spreading of computer viruses on time-varying networks," Physical Review E, vol. 99, no. 5, Article ID 050303, 2019.

[10] N. Sherborne, K. B. Blyuss, and I. Z. Kiss, "Bursting endemic bubbles in an adaptive network," Physical Review E, vol. 97, no. 4, Article ID 042306, 2018.

[11] H. Sayama, I. Pestov, J. Schmidt et al., "Modeling complex systems with adaptive networks," Computers \& Mathematics with Applications, vol. 65, no. 10, pp. 1645-1664, 2013.

[12] A. Szabó-Solticzky, L. Berthouze, I. Z. Kiss, and P. L. Simon, "Oscillating epidemics in a dynamic network model: stochastic and mean-field analysis," Journal of Mathematical Biology, vol. 72, no. 5, pp. 1153-1176, 2016.

[13] A. Szabó, "Dynamics of a link-type independent adaptive epidemic model," Differential Equations \& Applications, vol. 9, no. 1, pp. 105-122, 2017.

[14] A. Bodó and P. L. Simon, "Analytic study of bifurcations of the pairwise model for SIS epidemic propagation on an adaptive network," Differential Equations and Dynamical Systems, vol. 25, pp. 1-20, 2017.

[15] D. Juher, J. Ripoll, and J. Saldaña, "Outbreak analysis of an SIS epidemic model with rewiring," Journal of Mathematical Biology, vol. 67, no. 2, pp. 411-432, 2013.

[16] T. Gross, C. D. D'Lima, and B. Blasius, "Epidemic dynamics on an adaptive network," Physical Review Letters, vol. 96, no. 20, Article ID 208701, 2006.

[17] M. J. Keeling, D. A. Rand, and A. J. Morris, "Correlation models for childhood epidemics," Proceedings of the Royal Society of London. Series B: Biological Sciences, vol. 264, no. 1385 , pp. 1149-1156, 1997.

[18] I. Z. Kiss, J. C. Miller, and P. L. Simon, Mathematics of Epidemics on Networks. From Exact to Approximate Models, Springer, Cham, Switzerland, 2017.

[19] X. Zhang, C. Shan, Z. Jin, and H. Zhu, "Complex dynamics of epidemic models on adaptive networks," Journal of Differential Equations, vol. 266, no. 1, pp. 803-832, 2019. 
[20] Q. Wu, X. Fu, M. Small, and X.-J. Xu, "The impact of awareness on epidemic spreading in networks," Chaos: An Interdisciplinary Journal of Nonlinear Science, vol. 22, no. 1, Article ID 013101, 2012.

[21] D. Han, Q. Shao, D. Li, and M. Sun, "How the individuals' risk aversion affect the epidemic spreading," Applied Mathematics and Computation, vol. 369, Article ID 124894, 2020.

[22] W. Wang, "Epidemic models with nonlinear infection forces," Mathematical Biosciences and Engineering, vol. 3, no. 1, pp. 267-279, 2006.

[23] S. Ruan and W. Wang, "Dynamical behavior of an epidemic model with a nonlinear incidence rate," Journal of Differential Equations, vol. 188, no. 1, pp. 135-163, 2003.

[24] M. J. Keeling, "The effects of local spatial structure on epidemiological invasions," Proceedings of the Royal Society of London. Series B: Biological Sciences, vol. 266, no. 1421, pp. 859-867, 1999.

[25] P. van den Driessche and J. Watmough, "Reproduction numbers and sub-threshold endemic equilibria for compartmental models of disease transmission," Mathematical Biosciences, vol. 180, no. 1-2, pp. 29-48, 2002.

[26] C. Shan, Y. Yi, and H. Zhu, "Nilpotent singularities and dynamics in an SIR type of compartmental model with hospital resources," Journal of Differential Equations, vol. 260, no. 5, pp. 4339-4365, 2016.

[27] C. Castillo-Chavez and B. Song, "Dynamical models of tuberculosis and their applications," Mathematical Biosciences and Engineering, vol. 1, no. 2, pp. 361-404, 2004.

[28] Y. A. Kuznetsov, Elements of Applied Bifurcation Theory, Springer-Verlag, New York, NY, USA, 3rd edition, 2004.

[29] P. Yu, "Closed-form conditions of bifurcation points for general differential equations," International Journal of Bifurcation and Chaos, vol. 15, no. 4, pp. 1467-1483, 2005.

[30] J. Carr, Applications of Centre Manifold Theory, Springe, New York, NY, USA, 1981.

[31] W. K. Y. Dhooge and A. Govaerts, "A matlab package for numerical bifurcation analysis of odes," ACM Transactions on Mathematical Software), vol. 29, no. 2, pp. 141-164, 2003.

[32] Z. Lajmiri, R. Khoshsiar Ghaziani, and I. Orak, "Bifurcation and stability analysis of a ratio-dependent predator-prey model with predator harvesting rate," Chaos, Solitons \& Fractals, vol. 106, pp. 193-200, 2018.

[33] Y. A. Kuznetsov, H. G. E. Meijer, B. Al Hdaibat, and W. Govaerts, "Improved homoclinic predictor for BogdanovTakens bifurcation," International Journal of Bifurcation and Chaos, vol. 24, no. 4, Article ID 1450057, 2014.

[34] D. T. Gillespie, "A general method for numerically simulating the stochastic time evolution of coupled chemical reactions," Journal of Computational Physics, vol. 22, no. 4, pp. 403-434, 1976.

[35] J. R. C. Piqueira and C. M. Batistela, "Considering quarantine in the SIRA malware propagation model," Mathematical Problems in Engineering, vol. 2019, Article ID 6467104, 8 pages, 2019. 